\section{POCKET MATHEMATICS}

\section{The Coordinate Method}

By I. M. Gelfand, E. G. Glagoleva and A. A. Kirillov. (Primer 1.) Pp. xi+68. $75 s$.

\section{Functions and Graphs}

By I. M. Gelfand, E. G. Glagoleva and E. E. Schnol. (Primer 2.) Pp. vii +102 . 75s.

\section{Sequences and Combinatorial Problems}

By S. I. Gelfand, M. L. Gerver, A. A. Kirillov, N. N. Konstantinov and A. G. Kushnirenko. (Workbook 1.) Pp. vii +85 . $75 s$.

\section{Learn Limits Through Problems}

By S. I. Gelfand, M. L. Gerver, A. A. Kirillov, N. N. Konstantinov and A. G. Kushnirenko, (Workbook 2.) Pp. vii $+70.65 s$.

\section{Mathematical Problems}

An Anthology. By E. B. Dynkin, A. A. Molchanov, A. L. Rozental and A. K. Tolpygo. (Workbook 3.) Pp. $\mathrm{vii}+69$. $75 s$.

\section{Limits and Continuity}

By P. P. Korovkin. (Course 1.) Pp. vii + 125. 85s.

\section{Differentiation}

By P. P. Korovkin. (Course 2.) Pp. vii +86. $75 s$.

(Pocket Mathernatical Library.) Revised English edition; translated and edited by Richard A. Silverman. (Gordon and Breach: New York, London and Paris, May 1969.)

THERE are many texts dealing with school mathematics and introductory university mathematies; but none (in English) with quite the flavour of the present series of seven small books, translated from the Russian. The series consists of two primers, three workbooks and two courses. The primers are concerned with coordinates, functions and graphs, while the workbooks emphasize problems, especially involving sequences, combinatorics and limits. The two course books deal with limits, continuity and differentiation. All books are well written, with illustrations and worked examples, and every enthusiastic mathematics sixth-former may read them for pleasure and profit, and especially in order to be introduced to some of the problem solving encountered at this level in the Soviet Union.

One of the most interesting of the volumes is that entitled Sequences and Combinatorial Problems. Here, eightynine sample problems are listed and detailed solutions are given. There follows, without solution, a set of thirtyseven test problems. There are some unusual problems relating to arithmetic and geometric progressions, but the combinatorial probloms are unfortunately confined to problems of counting. The latter, however, may be due to the difficulty of constructing elementary structural problems of a combinatorial character with simple solutions. The volume entitled Mathematical Problems: An Anthology is a gem. It consists of 143 problems, of many different types, with hints and answers, and a set of thirtyfive supplementary problems. Some of the problems are tantalizing in their simplicity of statement. It may be of interest to quote two examples:

Problem 1.-A million lines are drawn in the plane such that no two lines are parallel and at loast one other line passes through the point of intersection of each pair of lines. Prove that all the lines are concurrent.

Problem 2.-Three rugs of arbitrary shape, each with an arca of 3 square yards, are spread on the floor of a room whose area is 6 square yards. Prove that two rugs overlap and share an area of at least one square yard.
In conclusion, it is worth recording a thought engendered by a reading of the books under consideration. It is clear that in elementary mathematics, as expounded in the Soviet Union, emphasis is still placed overwhelmingly on the classical concepts and sense of values (such as manipulation, but not for its own sake, and problem formulation and solution). It is still respectable to deal with counting problems involving permutations and combinations, and to ask and answer questions which involve an appeal to Euclidean geometry. In fact the biggest single surprise on reading the books is that of finding the degree of emphasis still placed on geometrical thinking. This retention of the "classical" mathematics rather than the wholesale acceptance of the so-called "new" mathematics is a sobering thought, which might well be followed by mathematicians in the western world, especially those concerned with the revision of school mathematics syllabuses.

\section{S. GODDARD}

\section{QUANTUM MECHANICS}

\section{Basic Quantum Mechanics}

By J. M. Cassels. (European Physics Series.) Pp. viii + 184. (MeGraw-Hill: London and Now York, 1970.) $62 s$ boards; $42 s$ paper.

IT is clear from the preface to this book that Professor Cassels endured all the usual agonies in learning quantum mechanics but was finally so impressed by the elegance and logic of Dirac's presentation that he is determined that his students shall not suffer the same fate. His solution is to present the subject as a set of concepts and rules followed by the logical consequences one may deduce for simple systems, but working throughout at a more elementary level than does Dirac. This seems almost certain to be unpopular with the older generation of physicists accustomed to the concept of the historical approach, but I must come down on the side of the author. If quantum mechanics always seems a difficult subject to teach to physics students, it is surely because the physical picture exists not in terms of elassical models but rather in the operator structure, so that the natural starting point must be with the language which works rather than with that which does not.

In general terms the book seems to achieve what was intended, the choice of topics being particularly good, but there remain a number of shortcomings. First, a rather high degree of physical background seems to be required for what is supposed to be a basic text. The reader must be presumed to have been oxposed to a fair amount of "the breakdown of classical physics", for no introduction of this type is incorporated. This seems particularly unfortunate, because such experience, insensitively given, could negate the whole concept of this style of presentation. Second, insufficient emphasis seems to have been given to the distinction between the basic quantum techniques and those approximations used in applications, so that there is a danger that students may confuse the two concepts. It is pleasing, however, to see such useful tools presented so early in such a basic course. Finally, there are no problems on which the student may test his comprehension. Exporience in teaching this subject convinces me that no student really understands the concepts until he has "got his hands dirty" himself.

Considered as a whole, this is a good book for a first course in non-relativistic quantum mechanics, but it should be adopted only by an experienced teacher who is convinced of the superiority of this method of presentation.

K. J. BARNES 